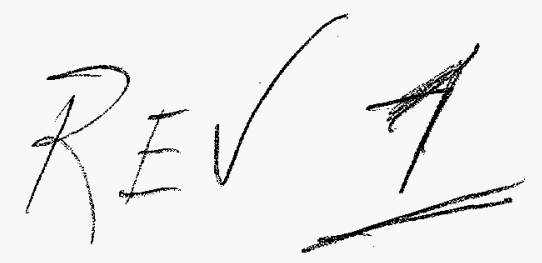

Periodicity of Crossover Currents in a Rutherford-Type Cable Subjected to a Time-Dependent Magnetic Field

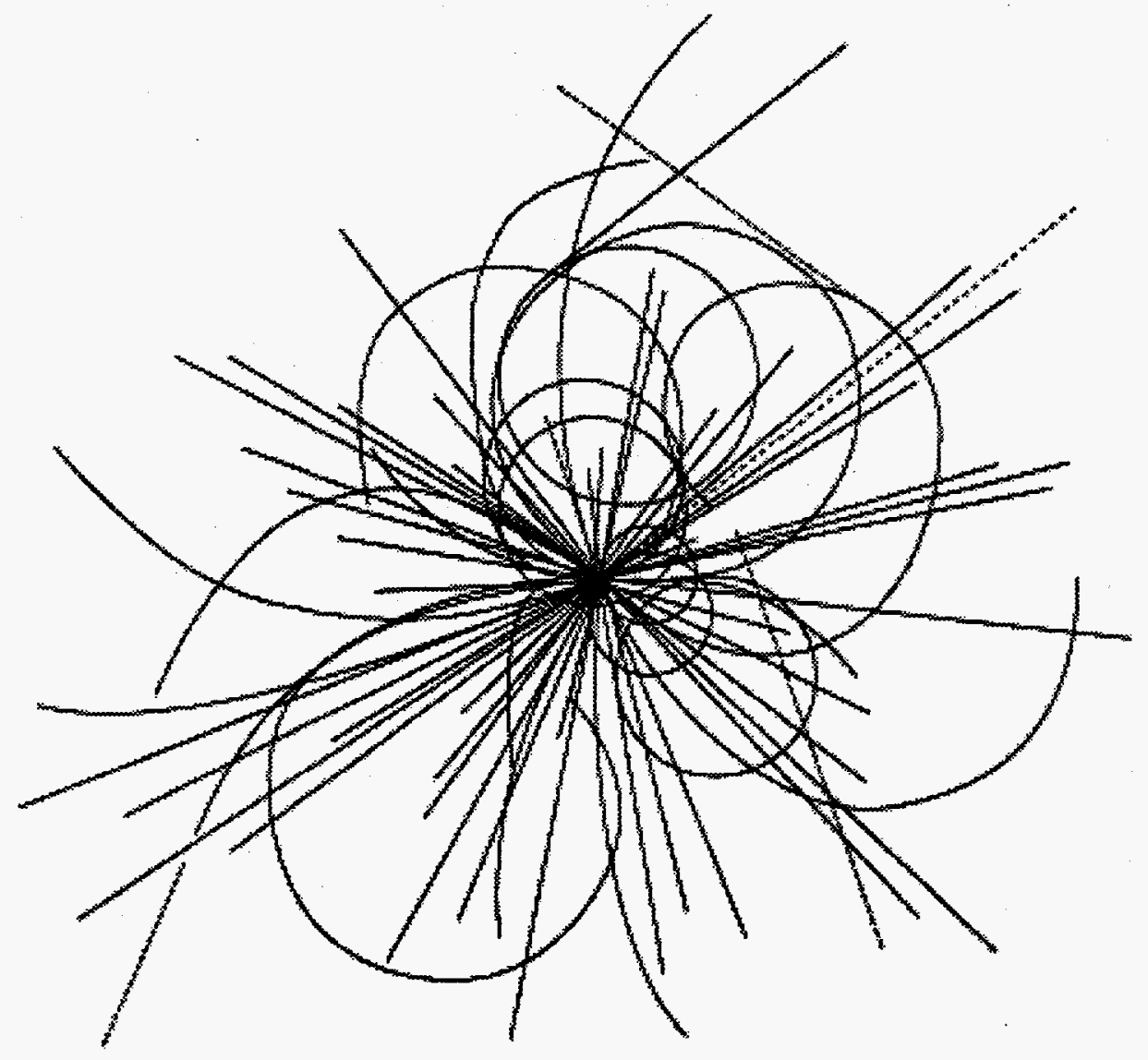

\section{Superconducting Super Collider Laboratory}

SSCL-Preprint-509 Rev. 1 September 1993

Distribution Category: 414
A. Akhmetov
A. Devred
T. Ogitsu 


\section{Disclaimer Notice}

This report was prepared as an account of work sponsored by an agency of the United States Govemment. Neither the United States Government or any agency thereof, nor any of their employees, makes any warranty, express or implied, or assumes any legal liability or responsibility for the accuracy, completeness, or uselulness of any information, apparatus, product, or process disclosed, or represents that its use would not intringe privately owned rights. Reference herein to any apectic commercial product, process, or service by trade name, trademark, manufacturer, or otherwise, does not necessarily constitute or imply its endorsement, recommendation, or favoring by the United States Govemment or any agency thereot. The views and opinions of authors expressed herein do not necessarily state or reflect thase of the United States Government or any egency thereot.

Superconducting Super Collider Laboratory is an equal opportunity employer. 


\title{
Periodicity of Crossover Currents in a Rutherford-Type Cable Subjected to a Time-Dependent Magnetic Field*
}

\author{
A. Akhmetov, ${ }^{1}$ A. Devred, ${ }^{1}$ and T. Ogitsu ${ }^{1,2}$ \\ ${ }^{1}$ Superconducting Super Collider Laboratory ${ }^{\dagger}$ \\ 2550 Beckleymeade Ave. \\ Dallas, TX 75237 \\ ${ }^{2}$ KEK, National Laboratory for High Energy Physics \\ 1-1 Oho, Tsukuba-shi \\ Ibaraki-ken, 305, Japan
}

September 1993

\footnotetext{
*Submitted to the Journal of Applied Physics.

TOperated by the Universities Research Association, Inc., for the U.S. Department of Energy under Contract No. DE-AC35-89ER40486.
} 


\section{DISCLAIMER}

Portions of this document may be illegible in electronic image products. Images are produced from the best available original document. 


\title{
Periodicity of crossover currents in a Rutherford-type cable subjected to a time-dependent magnetic field
}

\author{
A. A. Akhmetov, ${ }^{*}{ }^{*}$ A. Devred, ${ }^{1}$ and T. Ogitsu ${ }^{1,2}$ \\ ${ }^{1}$ Superconducting Super Collider Laboratory ${ }^{\dagger}$ \\ 2550 Beckleymeade Avenue \\ Dallas, TX 75237, USA \\ ${ }^{2}$ KEK, National Laboratory for High Energy Physics \\ 1-1 Oho, Tsukuba-shi \\ Ibaraki-ken, 305, Japan
}

\begin{abstract}
The behavior of Rutherford-type cables under a time-dependent magnetic field is studied. Existing models are extended describing the currents flowing through the resistive contacts at the crossovers between the cable strands by considering crossover current distributions which are not uniform along the cable axis. The generalized system of equations are applied to a few cases of practical interest and show that, if not uniform, the crossover current distribution is periodic, with a period equal to the cable pitch length.
\end{abstract}



$=$ 


\section{INTRODUCTION}

The response of homogeneous conductors to a time-dependent external magnetic field can be derived analytically only for simple geometries, such as infinite slabs or cylindrical bars. ${ }^{1}$ More elaborate geometries usually require the application of sophisticated numerical methods. ${ }^{2}$ The problem becomes more intricate when considering heterogeneous conductors, such as superconducting multifilamentary composites. These composites consist of a large number of superconducting filaments embedded in a matrix of normal metal. Here, a timedependent external field produces screening currents in both the superconducting filaments and the normal metal matrix. ${ }^{3}$ Over the years, a variety of models have been developed and numerous experimental studies have been carried out to determine the corresponding eddycurrent distributions. ${ }^{3-6}$

Another degree of complexity is added when considering the cables used in most of the superconducting magnets built today. These cables consist of a certain number of wires or strands, which are drawn from superconducting multifilamentary composite, and which are twisted or transposed together, as shown in Figure 1. The twisting or transposition results in a complicated network of superconducting paths (along the superconducting filaments of the wires) and of potentially low, resistive contacts, which are either distributed between adjacent twisted wires or localized at the points where transposed wires crossover one another. Through the resistive contacts, loops are formed between the cable strands where eddy currents can be generated when subjected to a time-dependent external field. ${ }^{7-9}$ These interstrand eddy currents are superimposed to the previously mentioned intra-strand eddy currents, which are produced within each individual strand. Such cable geometry has at least one property of symmetry: the network repeats itself after a certain distance along the cable axis, called the twist or transposition pitch length.

The present work deals with the so-called Rutherford-type conductors used in superconducting particle accelerator magnets. These conductors consist of a few tens of 
strands, twisted together, and shaped into a flat, two-layer, slightly keystoned cable. ${ }^{10,11} \mathrm{~A}$ model describing inter-strand eddy currents was first developed for braided conductors. ${ }^{7}$ The model was later extended to Rutherford-type conductors. ${ }^{12}$ In these references, however, the cable properties, the time-dependent external field and the screening currents, which were allowed to vary across the cable width, were assumed to be uniform along the cable length. This paper presents a generalization of the existing models to cases where the screening currents are not assumed to be uniform along the cable length.

\section{CONDUCTOR MODEL AND BASIC EQUATIONS}

Following references 7 and 12, the $N$-strand Rutherford-type cable shown in Figure 1 is described by the model circuit shown in Figure 2. In this model, the strands are assumed to be straight, except at the edges of the cable where they are bent in a hairpin-like manner to ramp from one layer to the other. Also, it is assumed that the only resistive contacts are at the crossovers between strands of the two layers. Thus, the smallest current loops, elementary loops, are constituted by two adjacent strands of one layer crossing over two adjacent strands of the other layer, as illustrated in Figure 3.

Two indexes are required to properly identify the crossover contacts of the model circuit in Figure 2: one for rows, $n$, where $1 \leq n \leq N-1$ and one for columns, $k$, where $k \geq 1$. A row is defined as a series of crossover contacts on a straight line parallel to the cable axis. A column is defined as a series of crossover contacts on a zigzag line across the cable width. The rows are counted starting from the thin edge of the slightly keystoned cable. The columns are counted from left to right, starting from an arbitrary position along the cable axis. The current circulating in a given crossover resistance, $r_{\mathrm{n}, \mathrm{k}}$, is referred to as $i_{\mathrm{n}, \mathrm{k}}$, and is counted positively when flowing from the bottom to the top layer of the cable (see Figure 3). The flux, $\Phi_{\mathrm{n}, \mathrm{k}}$, through an elementary loop is reckoned by the indexes of the crossover resistance at its left-hand-side corner and is counted positively when penetrating the cable 
from the bottom (see Figure 3). For a given column of a $N$-strand cable, there are $(N-1)$ crossover resistances, crossover currents and elementary loops.

To determine the crossover currents, apply Faraday's law to the $(N-1)$ elementary loops of the $k$-th column. For the loop at the cable thin edge, (see Figure 3(a)), we get

$$
r_{1, \mathrm{k}} i_{1, \mathrm{k}}+r_{1, \mathrm{k}+1} i_{1, \mathrm{k}+1}-r_{2, \mathrm{k}} i_{2, \mathrm{k}}=\frac{\mathrm{d} \Phi_{1, \mathrm{k}}}{\mathrm{d} t}
$$

For the loops in the middle of the cable (see Figure 3(b)), and for $n$ even, $2 \leq n \leq N-2$, we get

$$
r_{\mathrm{n}, \mathrm{k}} i_{\mathrm{n}, \mathrm{k}}+r_{\mathrm{n}, \mathrm{k}+1} i_{\mathrm{n}, \mathrm{k}+1}-r_{\mathrm{n}+1, \mathrm{k}+1} i_{\mathrm{n}+1, \mathrm{k}+1}-r_{\mathrm{n}-1, \mathrm{k}+1} i_{\mathrm{n}-1, \mathrm{k}+1}=\frac{\mathrm{d} \Phi_{\mathrm{n}, \mathrm{k}}}{\mathrm{d} t}
$$

while for $n$ odd, $3 \leq n \leq N-2$, we get

$$
r_{\mathrm{n}, \mathrm{k}} i_{\mathrm{n}, \mathrm{k}}+r_{\mathrm{n}, \mathrm{k}+1} i_{\mathrm{n}, \mathrm{k}+1}-r_{\mathrm{n}+1, \mathrm{k}} i_{\mathrm{n}+1, \mathrm{k}}-r_{\mathrm{n}-1, \mathrm{k}} i_{\mathrm{n}-1, \mathrm{k}}=\frac{\mathrm{d} \Phi_{\mathrm{n}, \mathrm{k}}}{\mathrm{d} t} .
$$

For the loop at the thick edge of the cable, and if $N$ is even, we get

$$
r_{\mathrm{N}-1, \mathrm{k}} i_{\mathrm{N}-1, \mathrm{k}}+r_{\mathrm{N}-1, \mathrm{k}+1} i_{\mathrm{N}-1, \mathrm{k}+1}-r_{\mathrm{N}-2, \mathrm{k}} i_{\mathrm{N}-2, \mathrm{k}}=\frac{\mathrm{d} \Phi_{\mathrm{N}-1, \mathrm{k}}}{\mathrm{d} t},
$$

while if $N$ is odd, we get

$$
r_{\mathrm{N}-1, \mathrm{k}} i_{\mathrm{N}-1, \mathrm{k}}+r_{\mathrm{N}-1, \mathrm{k}+1} i_{\mathrm{N}-1, \mathrm{k}+1}-r_{\mathrm{N}-2, \mathrm{k}+1} i_{\mathrm{N}-2, \mathrm{k}+1}=\frac{\mathrm{d} \Phi_{\mathrm{N}-1, \mathrm{k}}}{\mathrm{d} t} .
$$

The preceding equations express that the time-dependent magnetic fluxes induce electromagnetic forces that drive eddy currents through the resistive elements of the loops. The difference between Eq. (2) and Eq. (3) arises from the fact that the odd and even loops are positioned differently with respect to the columns of nodes. Eq. (1) is similar to Eq. (3), except that the bottom crossover resistance, $r_{\mathrm{n}-1, \mathrm{k}}$, is missing, while Eq. (4a) and Eq. (4b) 
are similar to Eq. (3) and Eq. (2), respectively, except that the top crossover resistances, $r_{\mathrm{n}+1, \mathrm{k}}$ or $r_{\mathrm{n}+1, \mathrm{k}+1}$, are missing. Conceptually, there is no difference between the case $N$ odd and $N$ even, except in the formulation of Eq. (4).

By combining Eqs. (1) through (Aa), it is possible to express the (N-1) crossover currents of the $(k+1)$-th column as linear combinations of the crossover currents and of the time-derivative of the elementary fluxes of the $k$-th column. For $N$ even,

$$
\begin{array}{r}
i_{1, \mathrm{k}+1}=-\frac{r_{1, \mathrm{k}}}{r_{1, \mathrm{k}+1}} i_{1, \mathrm{k}}+\frac{r_{2, \mathrm{k}}}{r_{1, \mathrm{k}+1}} i_{2, \mathrm{k}}+\frac{1}{r_{1, \mathrm{k}+1}} \frac{\mathrm{d} \Phi_{1, \mathrm{k}}}{\mathrm{d} t} \\
i_{2, \mathrm{k}+1}=-\frac{r_{1, \mathrm{k}}}{r_{2, \mathrm{k}+1}} i_{1, \mathrm{k}}+\frac{r_{2, \mathrm{k}}}{r_{2, \mathrm{k}+1}} i_{2, \mathrm{k}}-\frac{r_{3, \mathrm{k}}}{r_{2, \mathrm{k}+1}} i_{3, \mathrm{k}}+\frac{r_{4, \mathrm{k}}}{r_{2, \mathrm{k}+1}} i_{4, \mathrm{k}} \\
+\frac{1}{r_{2, \mathrm{k}+1}}\left(\frac{\mathrm{d} \Phi_{1, \mathrm{k}}}{\mathrm{d} t}+\frac{\mathrm{d} \Phi_{2, \mathrm{k}}}{\mathrm{d} t}+\frac{\mathrm{d} \Phi_{3, \mathrm{k}}}{\mathrm{d} t}\right)
\end{array}
$$

$$
i_{\mathrm{n}, \mathrm{k}+1}=\frac{r_{\mathrm{n}-1, \mathrm{k}}}{r_{\mathrm{n}, \mathrm{k}+1}} i_{\mathrm{n}-1, \mathrm{k}}-\frac{r_{\mathrm{n}, \mathrm{k}}}{r_{\mathrm{n}, \mathrm{k}+1}} i_{\mathrm{n}, \mathrm{k}}+\frac{r_{\mathrm{n}+1, \mathrm{k}}}{r_{\mathrm{n}, \mathrm{k}+1}} i_{\mathrm{n}+1, \mathrm{k}}+\frac{1}{r_{\mathrm{n}, \mathrm{k}+1}} \frac{\mathrm{d} \Phi_{\mathrm{n}, \mathrm{k}}}{\mathrm{d} t},
$$

for $n$ odd, $3 \leq n \leq N-2$,

$$
\begin{aligned}
\mathrm{n}, \mathrm{k}+1 & =\frac{r_{\mathrm{n}-2, \mathrm{k}}}{r_{\mathrm{n}, \mathrm{k}+1}} i_{\mathrm{n}-2, \mathrm{k}}-\frac{r_{\mathrm{n}-1, \mathrm{k}}}{r_{\mathrm{n}, \mathrm{k}+1}} i_{\mathrm{n}-1, \mathrm{k}}+\frac{\mathrm{m}_{\mathrm{n}, \mathrm{k}}}{r_{\mathrm{n}, \mathrm{k}+1}} i_{\mathrm{n}, \mathrm{k}}-\frac{r_{\mathrm{n}+1, \mathrm{k}}}{m_{\mathrm{m}, \mathrm{k}+1}} i_{\mathrm{n}+1, \mathrm{k}} \\
& +\frac{r_{\mathrm{n}+2, \mathrm{k}}}{r_{\mathrm{n}, \mathrm{k}+1}} i_{\mathrm{n}+2, \mathrm{k}}+\frac{1}{r_{\mathrm{n}, \mathrm{k}+1}}\left(\frac{\mathrm{d} \Phi_{\mathrm{n}-1, \mathrm{k}}}{\mathrm{d} t}+\frac{\mathrm{d} \Phi_{\mathrm{n}, \mathrm{k}}}{\mathrm{d} t}+\frac{\mathrm{d} \Phi_{\mathrm{n}+1, \mathrm{k}}}{\mathrm{d} t}\right),
\end{aligned}
$$

for $n$ even, $4 \leq n \leq N-4$,

$$
\begin{gathered}
i_{\mathrm{N}-2, \mathrm{k}+1}=\frac{r_{\mathrm{N}-4, \mathrm{k}}}{r_{\mathrm{N}-2, \mathrm{k}+1}} i_{\mathrm{N}-4, \mathrm{k}}-\frac{r_{\mathrm{N}-3, \mathrm{k}}}{r_{\mathrm{N}-2, \mathrm{k}+1}} i_{\mathrm{N}-3, \mathrm{k}}+\frac{r_{\mathrm{N}-2, \mathrm{k}}}{r_{\mathrm{N}-2, \mathrm{k}+1}} i_{\mathrm{N}-2, \mathrm{k}}-\frac{r_{\mathrm{N}-1, \mathrm{k}}}{r_{\mathrm{N}-2, \mathrm{k}+1}} i_{\mathrm{N}-1, \mathrm{k}} \\
+ \\
\quad \frac{1}{r_{\mathrm{N}-2, \mathrm{k}+1}}\left(\frac{\mathrm{d} \Phi_{\mathrm{N}-3, \mathrm{k}}}{\mathrm{d} t}+\frac{\mathrm{d} \Phi_{\mathrm{N}-2, \mathrm{k}}}{\mathrm{d} t}+\frac{\mathrm{d} \Phi_{\mathrm{N}-1, \mathrm{k}}}{\mathrm{d} t}\right), \\
i_{\mathrm{N}-1, \mathrm{k}+1}=\frac{r_{\mathrm{N}-2, \mathrm{k}}}{r_{\mathrm{N}-1, \mathrm{k}+1}} i_{\mathrm{N}-2, \mathrm{k}}-\frac{r_{\mathrm{N}-1, \mathrm{k}}}{r_{\mathrm{N}-1, \mathrm{k}+1}} i_{\mathrm{N}-1, \mathrm{k}}+\frac{1}{r_{\mathrm{N}-1, \mathrm{k}+1}} \frac{\mathrm{d} \Phi_{\mathrm{N}-1, \mathrm{k}}}{\mathrm{d} t} .
\end{gathered}
$$

$-6-$ 
A similar set of equations can be derived for $N$ odd. It follows from these equations that if the $(N-1)$ crossover currents of a given column of the cable are known, the crossover currents of the subsequent columns can be deduced by recurrence.

Below are a few simplified cases using the previously defined equations.

\section{MORGAN'S SOLUTION FOR THE CASE OF UNIFORM CROSSOVER RESISTANCE AND UNIFORM MAGNETIC FLUX}

Consider the case where the crossover resistance and the elementary magnetic flux vary across the cable width, but are uniform along the cable axis. Solve for the solutions of Eq. (5) satisfying the following equation:

$$
i_{\mathrm{n}, \mathrm{k}+1}=i_{\mathrm{n}, \mathrm{k}}, \quad \text { for } n, 1 \leq n \leq N-1 .
$$

In this case, the dependence of Eqs. (5a) through (5f) on the column number vanishes, and the system can be solved analytically:

$$
i_{1}=\frac{1}{N r_{1}} \sum_{p=1}^{N-1}(N-p) \frac{\mathrm{d} \Phi_{\mathrm{p}}}{\mathrm{d} t},
$$

and

$$
i_{\mathrm{n}}=n \frac{r_{1}}{r_{\mathrm{n}}} i_{1}-\frac{1}{r_{\mathrm{n}}} \sum_{p=1}^{n-1}(n-p) \frac{\mathrm{d} \Phi_{\mathrm{p}}}{\mathrm{d} t}, \quad \text { for } n, 2 \leq n \leq N-1
$$

(Note that Eqs. (7a) and (7b) apply for both $N$ even and $N$ odd.)

The power, $W$, dissipated by the crossover currents per cable unit length is given by the following equation:

$$
W=\frac{N}{2 L} \sum_{n=1}^{N-1} r_{\mathrm{n}} i_{\mathrm{n}}^{2}
$$

where $L$ is the cable half pitch length. 
Furthermore, assuming that the crossover resistances and the elementary fluxes are all equal, Eqs. (7) and (8) become

$$
i_{\mathrm{n}}=\frac{n(N-n)}{2 r} \frac{\mathrm{d} \Phi}{\mathrm{d} t}, \quad \text { for } n, 1 \leq n \leq N-1 .
$$

and

$$
W=\frac{N^{2}\left(N^{4}-1\right)}{240 L r}\left(\frac{\mathrm{d} \Phi}{\mathrm{d} t}\right)^{2}
$$

Considering that there are $N(N-1)$ elementary loops per cable pitch length, an estimate of $\Phi$ is simply given by the following:

$$
\Phi \approx \frac{2 h L}{N(N-1)} B
$$

where $h$ is the cable width, and $B$ is the supposedly uniform component of the magnetic flux density perpendicular to the cable. Combining Eqs. (10) and (11) results in the following:

$$
W \approx \frac{\left(N^{4}-1\right) h^{2} L}{60(N-1)^{2} r}\left(\frac{\mathrm{d} B}{\mathrm{~d} t}\right)^{2} \approx \frac{N^{2} h^{2} L}{60 r}\left(\frac{\mathrm{d} B}{\mathrm{~d} t}\right)^{2},
$$

where we recognize the expression given by Morgan. ${ }^{7}$

It thus appears that in the case of uniform crossover resistance and uniform magnetic flux along the cable axis, Eq. (5) does have a solution which satisfies Eq. (6), i.e., such that the crossover currents are the same in every column of the cable. In practice, however, Eq. (5) has to be solved along with a set of boundary conditions, which, for a given column of the cable, may impose values of crossover currents which are different from that of Eq. (7). Then the problem has to be reconsidered, and a more general solution has to be determined. 


\section{GENERAL SOLUTION FOR THE CASE OF UNIFORM CROSSOVER RESISTANCE AND UNIFORM MAGNETIC FLUX}

\section{Basic Equations}

Consider the case where all the crossover resistances are equal and where the elementary fluxes are uniform along the cable axis. For the sake of simplicity, also assume that $N$ is even and $M=N / 2$ is odd, and that the elementary fluxes are symmetrical with respect to the cable center line, i.e.,

$$
\Phi_{\mathrm{N}-\mathrm{n}}=\Phi_{\mathrm{n}} \quad \text { for } \mathrm{n}, 1 \leq n \leq M .
$$

Also, limiting the search for the solutions which satisfy

$$
i_{\mathrm{N}-\mathrm{n}, \mathrm{k}}=i_{\mathrm{n}, \mathrm{k}}, \quad \text { for } n, 1 \leq n \leq M,
$$

Eq. (5) can be reduced to the following system of $M$ equations:

$$
\begin{gathered}
i_{1, \mathrm{k}+1}=-i_{1, \mathrm{k}}+i_{2, \mathrm{k}}+\frac{1}{r} \frac{\mathrm{d} \Phi_{1}}{\mathrm{~d} t}, \\
i_{2, \mathrm{k}+1}=-i_{1, \mathrm{k}}+i_{2, \mathrm{k}}-i_{3, \mathrm{k}}+i_{4, \mathrm{k}}+\frac{1}{r}\left(\frac{\mathrm{d} \Phi_{1}}{\mathrm{~d} t}+\frac{\mathrm{d} \Phi_{2}}{\mathrm{~d} t}+\frac{\mathrm{d} \Phi_{3}}{\mathrm{~d} t}\right), \\
\ldots \quad \\
i_{\mathrm{n}, \mathrm{k}+1}=i_{\mathrm{n}-1, \mathrm{k}}-i_{\mathrm{n}, \mathrm{k}}+i_{\mathrm{n}+1, \mathrm{k}}+\frac{1}{r} \frac{\mathrm{d} \Phi_{\mathrm{n}}}{\mathrm{d} t}, \\
\text { for } n \text { odd, } 3 \leq n \leq M-2, \\
i_{\mathrm{n}, \mathrm{k}+1}=i_{\mathrm{n}-2, \mathrm{k}}-i_{\mathrm{n}-1, \mathrm{k}}+i_{\mathrm{n}, \mathrm{k}}-i_{\mathrm{n}+1, \mathrm{k}}+i_{\mathrm{n}+2, \mathrm{k}} \\
+\frac{1}{r}\left(\frac{\mathrm{d} \Phi_{\mathrm{n}-1}}{\mathrm{~d} t}+\frac{\mathrm{d} \Phi_{\mathrm{n}}}{\mathrm{d} t}+\frac{\mathrm{d} \Phi_{\mathrm{n}+1}}{\mathrm{~d} t}\right),
\end{gathered}
$$


for $n$ even, $4 \leq n \leq M-3$,

$$
\begin{aligned}
& i_{\mathrm{M}-1, \mathrm{k}+1}=i_{\mathrm{M}-3, \mathrm{k}}- i_{\mathrm{M}-2, \mathrm{k}}+2 i_{\mathrm{M}-1, \mathrm{k}}-i_{\mathrm{M}, \mathrm{k}} \\
&+\frac{1}{r}\left(\frac{\mathrm{d} \Phi_{\mathrm{M}-2}}{\mathrm{~d} t}+\frac{\mathrm{d} \Phi_{\mathrm{M}-1}}{\mathrm{~d} t}+\frac{\mathrm{d} \Phi_{\mathrm{M}}}{\mathrm{d} t}\right), \\
& i_{\mathrm{M}, \mathrm{k}+1}=2 i_{\mathrm{M}-1, \mathrm{k}}-i_{\mathrm{M}, \mathrm{k}}+\frac{1}{r} \frac{\mathrm{d} \Phi_{\mathrm{M}}}{\mathrm{d} t}
\end{aligned}
$$

\section{Matrix Notation}

The above system can be arranged into a more compact form by using matrix notation. Let $\mathbf{i}_{\mathbf{k}}$ and $\Phi$ designate the $M$-line, 1-column matrices, defined as

$$
\mathbf{i}_{\mathrm{k}}=\left[\begin{array}{c}
i_{1, \mathrm{k}} \\
i_{2, \mathrm{k}} \\
i_{3, \mathrm{k}} \\
i_{4, \mathrm{k}} \\
\\
\cdots \\
i_{\mathrm{n}, \mathrm{k}} \\
i_{\mathrm{n}+1, \mathrm{k}} \\
\\
\cdots \\
i_{\mathrm{M}-2, \mathrm{k}} \\
i_{\mathrm{M}-1, \mathrm{k}} \\
i_{\mathrm{M}, \mathrm{k}}
\end{array}\right]
$$$$
\Phi=\left[\begin{array}{c}
\phi_{1} \\
\phi_{1}+\phi_{2}+\phi_{3} \\
\phi_{3} \\
\phi_{3}+\phi_{4}+\phi_{5} \\
\ldots \\
\phi_{\mathrm{n}}+\phi_{\mathrm{n}+1}+\phi_{\mathrm{n}+2} \\
\phi_{\mathrm{n}} \\
\phi_{\mathrm{M}-2} \\
\phi_{\mathrm{M}-2}+\phi_{\mathrm{M}} \\
\phi_{\mathrm{M}}
\end{array}\right]
$$ 
and let $\mathbf{A}$ designate the square matrix of order $M$ :

$$
\begin{aligned}
& \mathbf{A}= \\
& {\left[\begin{array}{ccccccccccccccccccc}
-1 & 1 & 0 & 0 & 0 & 0 & \cdots & 0 & 0 & 0 & 0 & 0 & 0 & 0 & \cdots & 0 & 0 & 0 & 0 \\
-1 & 1 & -1 & 1 & 0 & 0 & \cdots & 0 & 0 & 0 & 0 & 0 & 0 & 0 & \cdots & 0 & 0 & 0 & 0 \\
0 & 1 & -1 & 1 & 0 & 0 & \cdots & 0 & 0 & 0 & 0 & 0 & 0 & 0 & \cdots & 0 & 0 & 0 & 0 \\
0 & 1 & -1 & 1 & -1 & 1 & \cdots & 0 & 0 & 0 & 0 & 0 & 0 & 0 & \cdots & 0 & 0 & 0 & 0 \\
\cdots & & & & & & & & & & & & & & & & & & \cdots \\
0 & 0 & 0 & 0 & 0 & 0 & \cdots & 0 & 1 & -1 & 1 & 0 & 0 & 0 & \cdots & 0 & 0 & 0 & 0 \\
0 & 0 & 0 & 0 & 0 & 0 & \cdots & 0 & 1 & -1 & 1 & -1 & 1 & 0 & \cdots & 0 & 0 & 0 & 0 \\
\cdots & & & & & & & & \cdots & & & & & & & & & & \cdots \\
0 & 0 & 0 & 0 & 0 & 0 & \cdots & 0 & 0 & 0 & 0 & 0 & 0 & 0 & \cdots & 1 & -1 & 1 & 0 \\
0 & 0 & 0 & 0 & 0 & 0 & \cdots & 0 & 0 & 0 & 0 & 0 & 0 & 0 & \cdots & 1 & -1 & 2 & -1 \\
0 & 0 & 0 & 0 & 0 & 0 & \cdots & 0 & 0 & 0 & 0 & 0 & 0 & 0 & \cdots & 0 & 0 & 2 & -1
\end{array}\right] .}
\end{aligned}
$$

With these notations, Eq. (15) can be rewritten:

$$
\mathbf{i}_{k+1}=A i_{k}+\frac{1}{r} \frac{d \Phi}{d t},
$$

where it clearly appears how the crossover currents of the $(k+1)$-th column can be derived from the crossover currents of the $k$-th column.

\section{Periodicity of Solution}

Apply Eq. (19) recurrently. The values of the crossover currents of the $(k+2)$-th column are:

$$
i_{k+2}=A i_{k+1}+\frac{1}{r} \frac{d \Phi}{d t}=A^{2} i_{k}+\frac{1}{r}\left(A+I_{d}\right) \frac{d \Phi}{d t},
$$

where $\mathbf{I}_{\mathbf{d}}$ is the identity matrix of order $M$. 
Similarly, for the crossover currents of the $(k+p)$-th column the values are

$$
\mathbf{i}_{\mathbf{k}+\mathbf{p}}=\mathbf{A}^{\mathbf{p}} \mathbf{i}_{\mathbf{k}}+\frac{1}{r}\left(\mathbf{A}^{\mathbf{p}-1}+\mathbf{A}^{\mathbf{p}-2}+\ldots+\mathbf{A}+\mathbf{I}_{\mathbf{d}}\right) \frac{\mathrm{d} \Phi}{\mathrm{d} t}
$$

It is shown in the appendix that the matrix A defined by Eq. (18) is such that

$$
\mathbf{A}^{\mathbf{M}}=-\mathbf{I}_{\mathbf{d}} \quad \text { (22a) } \quad \text { and } \quad \mathbf{A}^{2 \mathbf{M}}=\mathbf{I}_{\mathbf{d}} \text {, }
$$

where $N=2 M$ is the total number of cable strands. Rewriting Eq. (21) for $p=2 M$ and combining it with Eq. (22b) gives

$$
\mathbf{i}_{k+2 M}=\mathbf{i}_{k}+\frac{1}{r}\left(A^{2 M-1}+A^{2 M-2}+\ldots+A+I_{d}\right) \frac{d \Phi}{d t} .
$$

Furthermore, from Eq. (22a) one has

$$
\mathbf{A}^{M}+\mathbf{I}_{\mathbf{d}}=\mathbf{0}
$$

where 0 designates the zero matrix of order $M$.

Multiply Eq. (24) by $\mathrm{A}^{\mathrm{s}}$

$$
A^{M+s}+A^{s}=0
$$

and sum Eq. (25) for $p$ between 1 and $M-1$ :

$$
\sum_{s=1}^{M-1} A^{M+s}+A^{s}=A^{2 M-1}+A^{2 M-2}+\ldots+A+I_{d}=0 .
$$

Combining Eqs. (26) and (23) results in the following:

$$
\mathbf{i}_{\mathbf{k}+2 \mathrm{M}}=\mathbf{i}_{\mathbf{k}} \text {. }
$$


Eq. (27) shows that the crossover currents of the $(k+N)$-th column are identical to the crossover currents of the $k$-th column, whatever the actual values of the magnetic fluxes are, providing that they are uniform along the cable axis. The solutions of Eq. (15) are thus periodic, with a period equal to $N$. In more practical terms, this means that the crossover current between any two strands of the cable is the same after every pitch length.

The only case considered is where $N$ is even and $M$ is odd, and where the magnetic fluxes and the crossover currents satisfy Eqs. (13) and (14). It can be shown either analytically or numerically that the preceding result also applies when there is no symmetry with respect to the cable center line, and it can be generalized to any case of parity of $M$ and $N$. The solutions of Eq. (5) for a $N$-strand Rutherford cable, whose crossover resistances are equal and which is subjected to a uniform magnetic flux along its axis, are thus always periodic, with a period equal to $N$.

As an illustration, the distribution of crossover currents along a 4-strand and a 30-strand Rutherford-type cable are shown in Figures 4(a) and 4(b). For these calculations some arbitrary values of crossover currents were set for the first column, and the time derivative of the elementary magnetic fluxes were assumed to be either zero, Fig. 4(a), or uniform across the cable width, Fig. 4(b). All the curves are periodic and the period coincides with the number of strands.

\section{Discussion}

Let us now go back to the case considered by Morgan, and determine the solution of Eq. (19) which satisfies Eq. (6):

$$
\mathbf{i}_{\mathbf{k}}=\mathbf{A} \mathbf{i}_{\mathbf{k}}+\frac{1}{r} \frac{\mathrm{d} \Phi}{\mathrm{d} t}
$$


which yields

$$
\mathbf{i}_{\mathbf{k}}=\frac{1}{r}\left(\mathbf{I}_{\mathbf{d}}-\mathbf{A}\right)^{-1} \frac{\mathrm{d} \Phi}{\mathrm{d} t}
$$

Of course, it can be verified that the solution given in Eq. (29) is identical to the solution given by Eq. (7) in the case of a constant crossover resistance.

Eq. (29) determines the only solution of Eq. (19) corresponding to a uniform distribution of crossover currents along the cable axis. If one column of the cable is such that the crossover currents are equal to the values given by Eq. (29), then, providing that $r$ and $\Phi$ do not change along the cable axis, the crossover currents in all the columns are the same. On the other hand, if there is one column of the cable for which the crossover currents are different from the values given by Eq. (29), then the crossover current distribution cannot be uniform. However, in this case, the crossover current distribution is periodic with a period equal to the cable pitch length.

Hence, for a Rutherford-type cable, whose crossover resistances are equal and which is subjected to a uniform magnetic flux along its axis, the crossover current distribution can be either uniform or periodic. In practice, the type of solution which takes place is determined by the boundary conditions at the cable ends. For cables of finite length, the boundary conditions are likely to force the crossover currents of the first and last columns to be different from the values given by Eq. (29), and the solutions are thus likely to be nonuniform. (Note: if the crossover current distribution is periodic, so are the power dissipated by the crossover currents in the crossover resistances and the resultant heating of the conductor.) 


\section{CASE OF UNIFORM CROSSOVER RESISTANCE AND NONUNIFORM MAGNETIC FLUX}

So far, we only considered cases where the magnetic flux varied across the cable width, but was uniform along the cable axis. In magnets, the magnetic flux penetration through the cable also depends on the position along the cable. This is the case in the ends of a dipole or a quadrupole coil, where the turns come out of the coil straight section and turn around. Consequently, the distribution of crossover currents has to be determined using Eq. (5).

To understand what kind of crossover current distribution may result, once again consider the case where the crossover resistances are equal. Assume, however, that the magnetic flux is uniform across the cable width and that its time derivative varies along the cable axis according to curve 1 of Figure 5(a). This curve intends to simulate the actual magnetic flux profile seen by a half-turn (which consists of two ends and a straight section) of a long dipole or quadrupole coil. It can be divided into five regions: two regions of low and uniform magnetic flux, $\Phi_{\mathrm{e}}$, corresponding to the curved parts of the coil ends; two regions where the magnetic flux rises sharply, corresponding to the region where the turns enter the coil straight section; and one region of large and uniform magnetic flux, $\Phi_{\mathrm{ss}}$, corresponding to the coil straight section. In addition, assume that the crossover currents of the first column satisfy

$$
i_{\mathrm{n}, 1}=\frac{n(N-n)}{2 r} \frac{\mathrm{d} \Phi_{\mathrm{e}}}{\mathrm{d} t}, \quad \text { for } n, 1 \leq n \leq N-1,
$$

which corresponds to Morgan's solution for the end regions. By recurrently applying Eq. (5), it is now possible to calculate the crossover currents in all the columns of the cable.

Curves 2 and 3 of Figure 5(a) show the results of such a computation for the first and second row of a 4-strand cable. As expected, the crossover currents are uniform in the end regions, where Morgan's solution applies. They rise sharply in the intermediate regions, 
where the time derivative of the flux changes rapidly, and they appear to be periodic, with a period equal to 4 , in the central region corresponding to the coil straight section. This example clearly illustrates how an axial change in the magnetic flux forces the solution of Eq. (5) to switch from Morgan's solution to a periodic solution.

Figure 5(b) presents a similar set of plots for a different profile of the time derivative of the magnetic flux. In this case, it is assumed that the time derivative of the flux increases gradually along the cable axis, and there is no central plateau. As for Figure 5(a), as soon as the time derivative of the flux starts to change, the crossover currents seemingly follow and their distribution begins to exhibit characters of pseudo-periodicity.

The structure of Eq. (5) suggests that long and smooth nonuniformity of crossover resistance along the cable axis produces effects similar to that caused by nonuniformities in the magnetic flux, forcing the crossover current distribution to switch from uniform to pseudo-periodic as soon as the crossover resistance starts to change. Such kinds of nonuniformities are known to exist in the case in dipole or quadrupole coils, where the crossover resistances can be shown to vary from turn to turn. ${ }^{12}$

\section{CONCLUSION}

A model was developed to determine the distribution of screening currents in a Rutherford-type cable subjected to a time-dependent magnetic field. It was shown that, for any section of the cable along which the magnetic flux and the resistive contacts at the crossovers between the cable strands are uniform, the currents induced in the crossover resistances are periodic with a period equal to the cable pitch length. Following Morgan it was reestablished that with the same assumptions and for a given profile of magnetic flux across the cable width, there is one degenerated solution for which the crossover currents are uniform along the cable axis. This solution, however, can only take place if the cable is infinite or if the crossover currents at a given axial position of the cable are forced to assume 
the appropriate values. Even if the degenerated solution is imposed on part of the cable, the occurrence of a nonuniformity in the magnetic flux or the crossover resistance forces the crossover current distribution to become periodic or pseudo-periodic. 


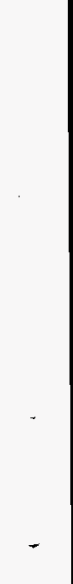




\section{APPENDIX}

Let us demonstrate by double recurrence on $M$, where $M$ is odd, that the characteristic polynomial, $P_{\mathrm{M}}$, of the square matrix $A$ of order $M$ defined by Eq. (18) is

$$
P_{M}(\lambda)=\operatorname{det}\left(\mathbf{A}-\lambda \mathbf{I}_{\mathbf{d}}\right)=-\lambda^{M}-1 \quad \text { for } \lambda, \lambda \in \mathrm{C}
$$

First verify this proposition for $M=3$ and $M=5$. For $M=3$, Eq. (18) becomes

$$
\mathbf{A}=\left[\begin{array}{ccc}
-1 & 1 & 0 \\
-1 & 2 & -1 \\
0 & 2 & -1
\end{array}\right]
$$

resulting in the following:

$$
P_{3}(\lambda)=\operatorname{det}\left[\begin{array}{ccc}
-1-\lambda & 0 & 0 \\
-1 & 2-\lambda & -1 \\
0 & 2 & -1-\lambda
\end{array}\right]=-\lambda^{3}-1
$$

Similarly, a direct calculation allows one to verify that, for $M=5$,

$$
P_{5}(\lambda)=-\lambda^{5}-1
$$

Now assume that the proposition is true for matrices $A$ of orders $M-2$ and $M$ :

$$
P_{\mathrm{M}-2}(\lambda)=-\lambda^{\mathrm{M}-2}-1
$$

and

$$
P_{\mathrm{M}}(\lambda)=-\lambda^{\mathrm{M}}-1
$$

and show that it implies that the proposition is also true for matrix $A$ of order $M+2$. 
Using Eq. 18 results in the following:

$P_{\mathrm{M}+2}=$
$\operatorname{dec}\left[\begin{array}{cccccccccccc}-1-\lambda & 1 & 0 & 0 & 0 & 0 & 0 & \cdots & 0 & 0 & 0 & 0 \\ -1 & 1-\lambda & -1 & 1 & 0 & 0 & 0 & \cdots & 0 & 0 & 0 & 0 \\ 0 & 1 & -1-\lambda & 1 & 0 & 0 & 0 & \cdots & 0 & 0 & 0 & 0 \\ 0 & 1 & -1 & 1-\lambda & -1 & 1 & 0 & \cdots & 0 & 0 & 0 & 0 \\ 0 & 0 & 1 & -1 & 1-\lambda & 0 & 0 & \cdots & 0 & 0 & 0 & 0 \\ 0 & 0 & 1 & -1 & 1 & -1-\lambda & 1 & \cdots & 0 & 0 & 0 & 0 \\ & & & & & & & & & & & \\ \cdots & & & & & & & & & & & \cdots \\ 0 & 0 & 0 & 0 & 0 & 0 & 0 & \cdots & 1 & -1-\lambda & 1 & 0 \\ 0 & 0 & 0 & 0 & 0 & 0 & 0 & \cdots & 1 & -1 & 2-\lambda & -1 \\ 0 & 0 & 0 & 0 & 0 & 0 & 0 & \cdots & 0 & 0 & 2 & -1-\lambda\end{array}\right]$.

By decomposing the above determinant along the first line, $P_{\mathrm{M}+2}$ can be expressed as a linear combination of determinants of order $(M+1)$. In turn, the determinants of order $(M+1)$ can be decomposed along either their first line or their first column and be expressed as linear combinations of determinants of order M. Reiterating the process two more times, it can be shown that

$$
P_{\mathrm{M}+2}(\lambda)=\lambda^{2} P_{\mathrm{M}}(\lambda)-(1+\lambda)\left[P_{\mathrm{M}-2}^{(1)}(\lambda)+P_{\mathrm{M}-2}^{(2)}(\lambda)\right],
$$


where $P_{\mathrm{M}-2}^{(1)}$ and $P_{\mathrm{M}-2}^{(2)}$ are two determinants of order $(M-2)$ defined as

$$
P_{M-2}^{(1)}(\lambda)=\operatorname{det}\left[\begin{array}{ccccccccc}
1 & 1 & 0 & 0 & \cdots & 0 & 0 & 0 & 0 \\
1 & 1-\lambda & -1 & 1 & \cdots & 0 & 0 & 0 & 0 \\
0 & 1 & -1-\lambda & 1 & \cdots & 0 & 0 & 0 & 0 \\
\cdots & & & & & & & & \cdots \\
0 & 0 & 0 & 0 & \cdots & 1 & -1-\lambda & 1 & 0 \\
0 & 0 & 0 & 0 & \cdots & 1 & -1 & 2-\lambda & -1 \\
0 & 0 & 0 & 0 & \cdots & 0 & 0 & 2 & -1-\lambda
\end{array}\right]
$$

and

$$
P_{M-2}^{(2)}(\lambda)=\operatorname{det}\left[\begin{array}{ccccccccc}
1 & -1-\lambda & 0 & 0 & \cdots & 0 & 0 & 0 & 0 \\
1 & -1 & -1 & 1 & \cdots & 0 & 0 & 0 & 0 \\
0 & 1 & -1-\lambda & 1 & \cdots & 0 & 0 & 0 & 0 \\
\cdots & & & & & & & & \cdots \\
0 & 0 & 0 & 0 & \cdots & 1 & -1-\lambda & 1 & 0 \\
0 & 0 & 0 & 0 & \cdots & 1 & -1 & 2-\lambda & -1 \\
0 & 0 & 0 & 0 & \cdots & 0 & 0 & 2 & -1-\lambda
\end{array}\right]
$$

To determine $P_{\mathrm{M}-2}^{(1)}$ and $P_{\mathrm{M}-2}^{(2)}$, decompose $P_{\mathrm{M}}$ following a process similar to the one described for $P_{\mathrm{M}+2}$. After two iterations, the result is

$$
P_{\mathrm{M}}(\lambda)=-(1+\lambda)\left[P_{\mathrm{M}-2}^{(1)}(\lambda)+P_{\mathrm{M}-2}^{(2)}(\lambda)\right]+\lambda^{2} P_{\mathrm{M}-2}(\lambda)
$$


Let us now combine Eqs. (A6) and (A9):

$$
P_{\mathrm{M}+2}(\lambda)=\lambda^{2}\left[P_{\mathrm{M}}(\lambda)-P_{\mathrm{M}-2}(\lambda)\right]+P_{\mathrm{M}}(\lambda)
$$

This equation shows how the characteristic polynomial of the matrix of order $(M+2)$ is related to the characteristic polynomials of the matrices of order $M$ and $(M-2)$. Finally, replacing $P_{\mathrm{M}}$ and $P_{\mathrm{M}-2}$ by their assumed expressions gives

$$
P_{\mathrm{M}+2}(\lambda)=-\lambda^{\mathrm{M}+2}-1
$$

where Eq. (A1) is recognized as written for a matrix of the order $(M+2)$.

We verified that Eq. (A1) is true for $M=3$ and $M=5$, and verified that if Eq. (A1) is true for matrices of order $M$ and $(M-2)$, it implies that it is also true for matrices of order $(M+2)$. Hence, Eq. (A1) is true for all $M$ odd.

It follows from Eq. (A1) that the eigenvalues of the square matrix $A$ of order $M$ are the solutions of the equation

$$
\lambda M+1=0,
$$

which correspond to the $M$-th roots of $(-1)$. The eigenvalues of the matrix $\mathbf{A}^{\mathbf{M}}$ are thus all equal to $(-1)$ and those of the matrix $A^{2 M}$ are all equal to 1 , and result in
$\mathbf{A}^{\mathbf{M}}=-\mathbf{I}_{\mathbf{d}}$
(A13a)
and
$\mathbf{A}^{2 M}=I_{d}$. 


\section{REFERENCES}

* Now at the Research Institute for Superconducting Magnets, Kyushu University, 610-1 Hakozaki, Hakozaki-ku, Fukuoka, 812, Japan.

$\dagger$ Operated by the University Research Association, Inc., for the U.S. Department of Energy under Contract No. DE-AC35-89ER40486.

1. R. M. Bozorth, Ferromagnetism, (Van Nostrand, Princeton, 1951), p. $769 \mathrm{ff}$.

2. J. J. H. Wang, Generalized Moment Methods of Electromagnetics, (John Wiley, New York, 1991), p. 553 ff.

3. M. N. Wilson, Superconducting Magnets (Oxford University Press, Oxford, 1983).

4. W. J. Carr, AC Loss and Macroscopic Theory of Superconductors, Gordon and Breach Science Publishers, New York, London, Paris, 1983).

5. V. A. Altov, V. B. Zenkevitch, M. G. Kremlev, and V. V. Sytchev, Stabilization of Superconducting Magnetic Systems, (Plenum, New York, 1973).

6. D. Ciazynski and B. Turck, "Theoretical and experimental study of the saturation of a superconducting composite under fast changing magnetic field," Cryogenics, 24, 507 (1984).

7. G. H. Morgan, "Eddy currents in flat metal filled superconducting braids," J. Appl. Phys., 44, 3319 (1973).

8. V. E. Sytnikov and G. G. Svalov, "Coupling losses in superconducting transposed conductors located in changing magnetic field," Cryogenics, 29, 926 (1989).

9. D. Ciazinsky, B. Turck, J. L. Duchateau, and C. Meuris, “AC losses and current distribution in $40 \mathrm{kA} \mathrm{NbTi}$ and $\mathrm{Nb}_{3} \mathrm{SN}$ conductors for NET/TTER," IEEE Trans. Appl. Super., 3, 594 (1993).

10. J. Royet and R. Scanlan, "Manufacture of Keystoned Flat Superconducting Cables for Use in SSC Dipoles," IEEE Trans. Mag., MAG-23, 480 (1987). 
11. D. Christoferson, D. Capone, R. Hannaford, R. Remsbottom, R. Delashmit, R. J. Jayakumar, G. Snitchler, R. Scanlan, and J. Royet, "SSC $40 \mathrm{~mm}$ cable results and 50 mm design discussions," IEEE Trans. Mag., MAG-27, 1881 (1991).

12. T. Ogitsu, Y. Zhao, A. Akhmetov, and A. Devred, "Influence of cable eddy currents on magnetic field harmonics," KEK-Proc. 92-14, KEK National Laboratory for High Energy Physics, 23 (1992). 


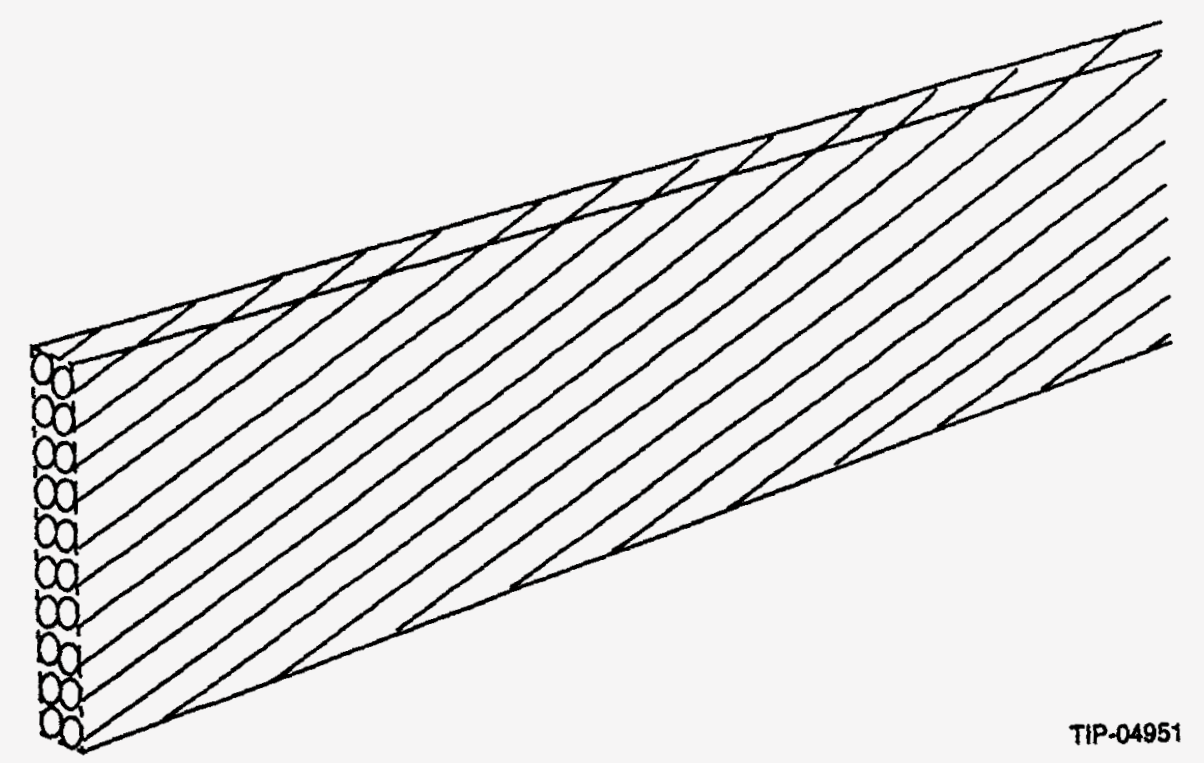

Figure 1. Rutherford-type cable used in superconducting particle accelerator magnets. 


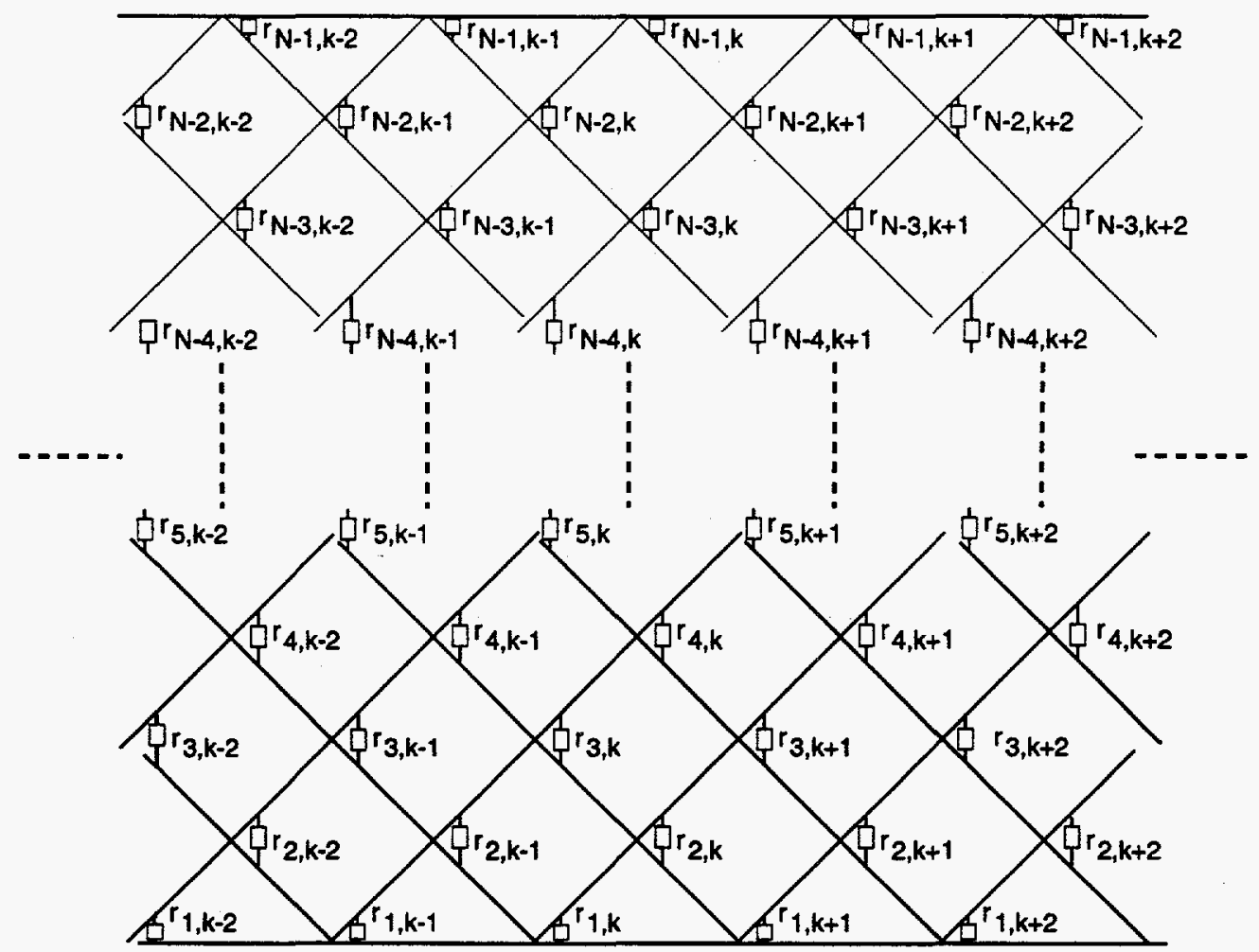

TIP-04952

Figure 2. Model circuit for a Rutherford-type cable subjected to a time-dependent magnetic field.

(a)

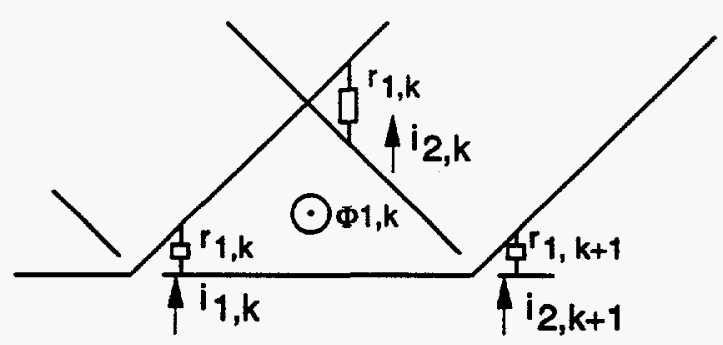

(b)

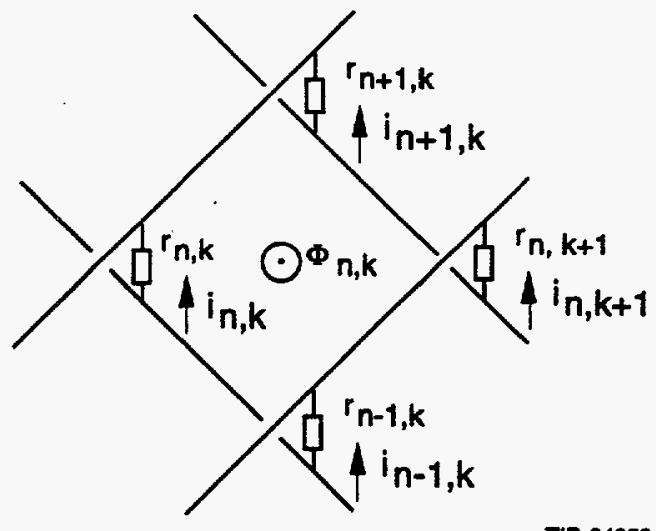

Figure 3. Elementary loops of the model circuit: (a) at the thin edge of the slightly keystoned cable, and (b) in the middle of the cable. 

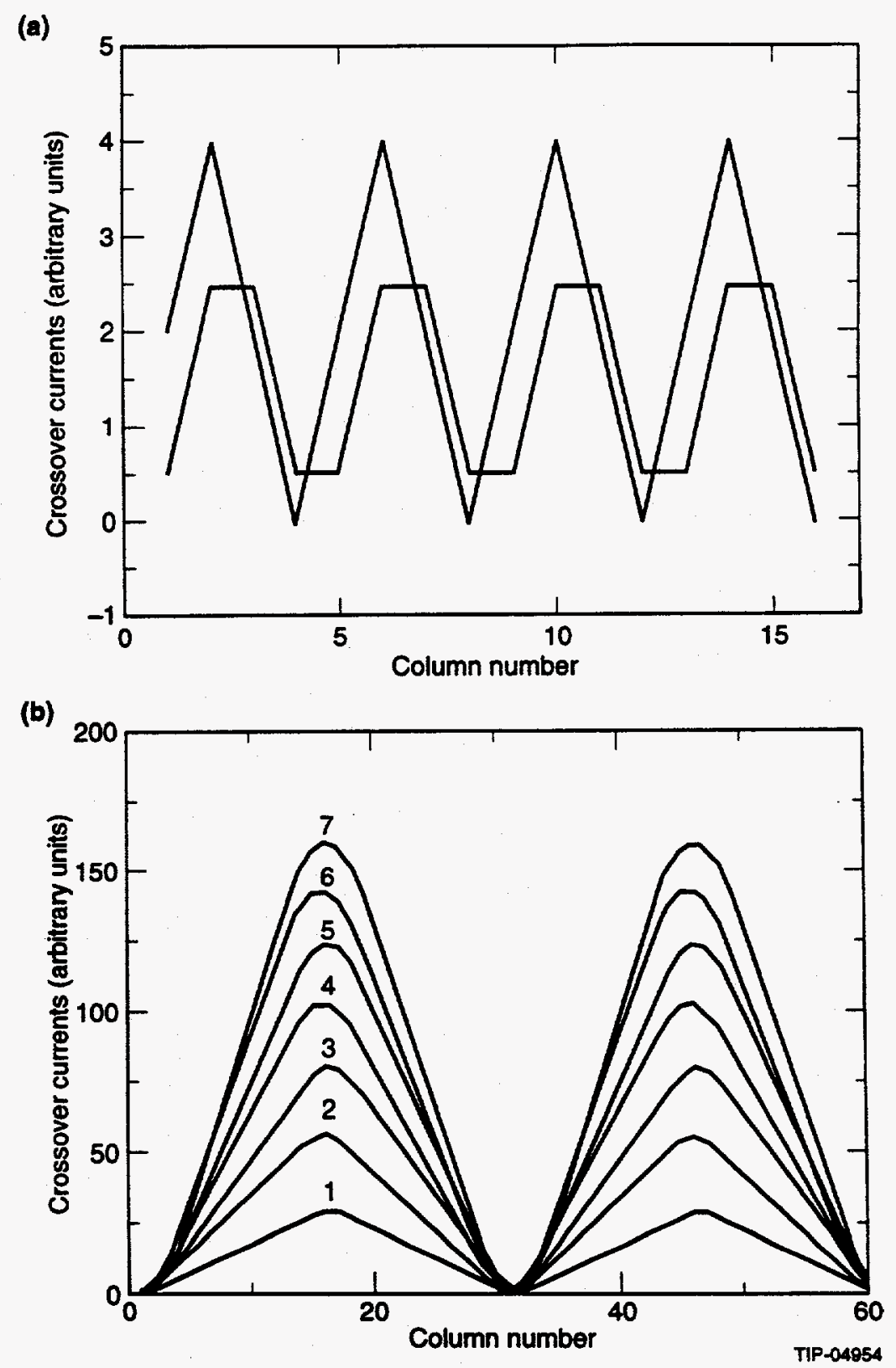

Figure 4. Distribution of crossover currents along a Rutherford-type cable subjected to a uniform, time-dependent magnetic field: (a) case of a 4-strand cable, and (b) case of a 30-strand cable. The crossover resistances are assumed to be equal. The curve numbers correspond to the cable row numbers. 

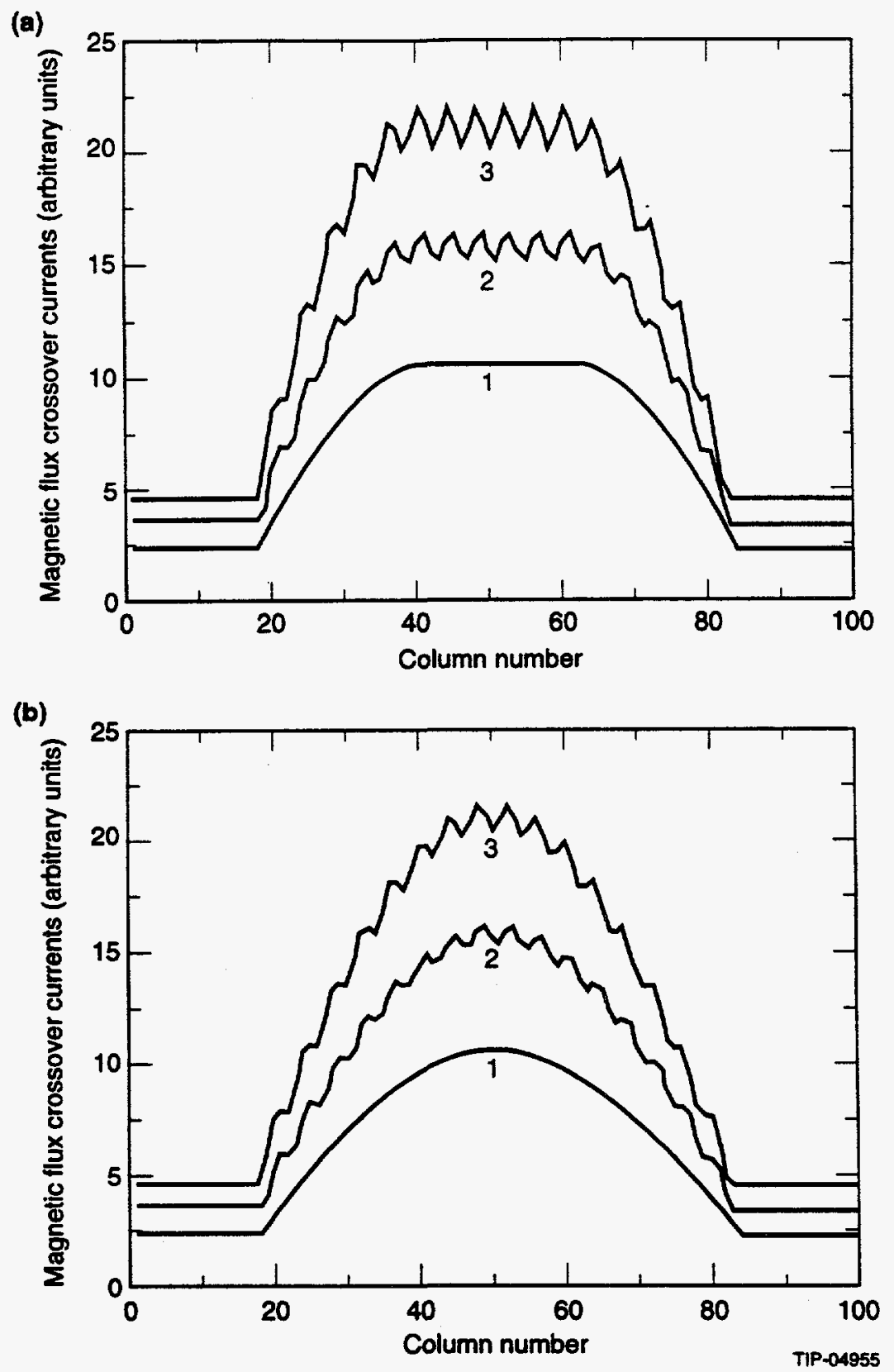

Figure 5(a) and (b). Distribution of crossover currents along a 4-strand Rutherford-type cable subjected to a nonuniform, time-dependent magnetic field. Curve (1) shows the assumed profile of the time-derivative of the magnetic flux. Curves (2) and (3) show the resulting crossover currents in the first and second rows of the cable. The crossover resistances are assumed to be equal. 